\title{
SCHOTTKY GROUPS AND LIMITS OF KLEINIAN GROUPS ${ }^{1}$
}

\author{
BY VICKI CHUCKROW
}

\author{
Communicated by Lipman Bers, August 15, 1966
}

Definition. A group of Möbius transformations is said to be a marked Schottky group of genus $g$ with standard generators $T_{1}, \cdots, T_{0}$, if there exist disjoint Jordan curves $\Gamma_{1}, \Gamma_{1}^{\prime}, \cdots, \Gamma_{g}, \Gamma_{g}^{\prime}$, which bound a $2 g$-times connected domain $D$, such that $T_{j}(D) \cap D=\varnothing$ and $T_{j}\left(\Gamma_{j}\right)=\Gamma_{j}^{\prime}, j=1, \cdots, g$.

LEMma 1. If $G$ is a Schottky group of genus $g$, then $G$ is a marked Schottky group on every set of $g$ free generators; i.e., every set of $g$ free generators for $G$ is standard.

The proof is based on the classical theorem on automorphisms of finitely generated free groups.

Lemma 2. Every finitely generated subgroup of a Schottky group is a Schottky group.

The proof uses the fact that all Schottky groups are quasi-conformally equivalent to certain Fuchsian groups.

REMARK. The preceding two lemmas can be generalized, with appropriate modifications, to Schottky type groups (see [1] for the definition).

Theorem 1. Let $T_{1}, \cdots, T_{g}$ be $g>1$ Möbius transformations. Suppose there exist marked Schottky groups of genus $g,\left\langle T_{1, n}, \cdots, T_{0, n}\right\rangle$, such that $\lim _{n \rightarrow \infty} T_{j, n}=T_{j}, j=1, \cdots$, g. Then the group $G$ generated by $T_{1}, \cdots, T_{g}$ is a free group on $g$ generators, without elliptic elements.

The proof uses Lemmas 1 and 2 and involves an elementary area argument.

Definition. An isomorphism $\phi: G_{1} \rightarrow G_{2}$ between two Kleinian groups is said to be type preserving if $\phi(T)$ is parabolic if and only if $T$ is.

Theorem 2. For every $n=0,1,2, \cdots$, let $G(n)=\left\{T_{j}(n)\right.$, $j=0,1, \cdots\}$ be a Kleinian group. Assume that there are Möbius transformations $T_{j}$ such that $\lim _{n \rightarrow \infty} T_{j}(n)=T_{j}$, and denote the group $\left\{T_{j}, j=0,1, \cdots\right\}$ by $G$. Assume also that all mappings $T_{j}(0) \rightarrow T_{j}(n)$

${ }_{1}^{1}$ This research was supported by a National Science Foundation Fellowship and constitutes a doctoral thesis. 
of $G(0)$ onto $G(n)$ are type preserving isomorphisms. Then the mapping $T_{j}(0) \rightarrow T_{j}$ is an isomorphism of $G(0)$ onto $G$, and $G$ contains no elliptic elements of infinite order.

The proof uses the observation of Myrberg that every Kleinian group contains Schottky subgroups, Theorem 1, and a recent result of Maskit [3].

Definition. We say that two marked Schottky groups $G=\left\langle T_{1}, \cdots, T_{a}\right\rangle$ and $G^{\prime}=\left\langle T_{1}^{\prime}, \cdots, T_{a}^{\prime}\right\rangle$ are equivalent if there exists a Möbius transformation $B$ such that $B T_{i} B^{-1}=T_{i}^{\prime}, i=1, \cdots, g$. The Schottky space of genus $g, S_{g}$, is the set of all equivalence classes of Schottky groups of genus $g>1$, with the natural topology.

There is a natural homeomorphism of $\operatorname{SL}^{\prime}(2, C)(\operatorname{SL}(2, C)$ factored by its center) onto $P_{3}(C)-X$, where $P_{3}(C)$ is complex projective 3space and $X$ the quadric corresponding to singular matrices. Thus the space of groups of Möbius transformations on $g$ generators, $\mathrm{SL}^{\prime}(2, \boldsymbol{C})^{d}$, is naturally homeomorphic to $\left(P_{3}(C)-X\right)^{d}$. Let $X_{0} \subset\left(P_{3}(\boldsymbol{C})-X\right)^{\circ}$ be the set of points which, considered as groups of Möbius transformations on $g$ generators, have at least three distinct fixed points for the $g$ generators. Every $B \in \operatorname{SL}^{\prime}(2, C)$ acts on $X_{0}$ by sending $G \in X_{0}$ into $B G B^{-1} \in X_{0}$. Let $\Lambda$ be the group of all such transformations. Then $X_{0} / \Lambda$ is a complex manifold of dimension $3 g-3$ and Schottky space $S_{o}$ is embedded as an open connected submanifold $S_{o}$ in $X_{0} / \Lambda$.

THEOREM 3. Every point on the boundary of $\hat{S}_{o}$ represents a free group on g generators, without elliptic elements.

This follows directly from Theorem 1.

Definition (cf. [2]). A point on the boundary of $\hat{S}_{g}$ is a cusp if the group it represents contains parabolic elements.

Theorem 4. A boundary point of $\hat{S}_{g}$ is either a cusp or represents a free strictly loxodromic group which is not Kleinian, i.e., not discontinuous.

The proof follows easily from a dimension argument, Theorem 1 , and a theorem of Maskit [3].

Acknowledgment. The author wishes to express her gratitude to her advisor Professor L. Bers.

\section{REFERENCES}

1. L. Ahlfors and L. Sario, Riemann surfaces, Princeton Univ. Press, Princeton, N. J., 1960. 
2. L. Bers and B. Maskit, On a class of Kleinian groups, Int. Symposium on Analytic Functions at Erevan (to appear).

3. B. Maskit, A characterization of Schottky groups, J. Analyse Math. (to appear). New York University, Courant Institute of Mathematical Sciences 